\title{
Applying harm reduction to smoking
}

\author{
John R Hughes
}

Harm reduction as applied to illicit drugs of dependence has included many different strategies (see Reuter and MacCoun, this issue, p. S28), some of which readily apply to smoking and some of which do not (for example, destigmatising use) (table). The idea of applying harm reduction strategies to smoking is not new and previous reviews of this notion have been published. ${ }^{1,2}$ Implementing such strategies could produce several benefits: decreased morbidity and mortality, decreased secondhand harm to non-users, decreased health care expenditure, and increased cessation. Implementing such strategies could also produce several problems: decreased cessation, increased initiation, and the diversion of time, money, and effort from research into and treatment for smoking cessation.

This article will focus on the use of safer delivery routes and decreasing drug intake as harm reducing strategies. Other papers in this volume (Orleans, p. S3 and Pinney, p. S10) discuss increasing access to treatment. For brevity, monitoring for associated diseases, protecting non-users, and destigmatising use will not be discussed.

\section{Less hazardous products: low tar, low nicotine cigarettes}

Because most low tar cigarettes reduce tar by dilution methods (for example, holes in the filters), most are of these are also low nicotine cigarettes. ${ }^{1,3}$ The most recent review of the benefits of low tar, low nicotine cigarettes was the President's Cancer Panel which concluded:

The smoking of cigarettes with lower machinemeasured yields has a small effect on reducing the risk of cancer caused by smoking, no effect on the risk of cardiovascular diseases and an uncertain effect on the risk of pulmonary disease. ${ }^{4}$

One of the reasons for the failure of the panel to state more resolutely that low tar, low nicotine cigarettes are beneficial was the absence of randomised trials of the effect of low versus high tar cigarettes on disease, or of longitudinal studies of the health outcomes of smokers who switch to low tar, low nicotine cigarettes. ${ }^{5}$ Another reason is that the positive data available compared smokers who smoked what are now considered very high tar cigarettes (for example, $>26 \mathrm{mg}$ tar/cigarette) with those who smoked what are now considered moderate tar cigarettes (for example, $<18 \mathrm{mg}$ tar/cigarette).$^{6,7}$ Whether the present low tar cigarettes which contain less than $6 \mathrm{mg}$ of tar offer additional benefit has not been investigated.

One often hypothesised reason for the small benefit from low tar cigarettes is that, if they are also low nicotine cigarettes, smokers will smoke more to obtain more nicotine and therebyoffsetany reductions in tar exposure. ${ }^{1-3,5}$ The increase in smoking can be accomplished through several behaviours: smoking more cigarettes per day, smoking more puffs per cigarette, and smoking each puff more deeply. However, the most important behaviour appears to be blocking of filter vents in cigarettes. ${ }^{1}$ Although the above compensatory behaviours clearly occur in short term studies, ${ }^{1,5,8}$ most long term studies suggest that such compensation does not completely negate the possible benefits of switching to low tar, low nicotine cigarettes. ${ }^{9-12}$ For example, one study of smokers three to six years after switching found a decline of $10 \mathrm{ppm}$ in carbon monoxide levels (figure 1). ${ }^{10}$

Although most people switch to low tar cigarettes on their own, there have been several studies using switching to low tar, low nicotine cigarettes as a preliminary to stopping smoking. These programmes typically have the subjects switch to gradually lower tar levels and monitor their progress graphically. The ability of these programmes to induce abstinence is mixed. ${ }^{13-19}$ Unfortunately, few of these studies reported on non-abstinence outcomes. In contrast to these studies, one study examined brand switching in smokers who did not wish to stop as part of a multifaceted controlled smoking programme. ${ }^{9,20}$ In this study, the average nicotine content per cigarette decreased from $0.8 \mathrm{mg} /$ cigarette before treatment to $0.4 \mathrm{mg} /$ cigarette after treatment and then increased slightly to $0.5 \mathrm{mg} /$ cigarette at a 2.5 year follow up. ${ }^{20}$

One concern about low tar, low nicotine cigarettes is their acceptability. Low tar cigarettes ( $<15 \mathrm{mg} /$ cigarette) have increased from less than $5 \%$ of the market to about $60 \%$ of

\section{Vermont Behavioral \\ Pharmacology \\ Laboratory, Depts of \\ Psychiatry, \\ Psychology, and \\ Family Practice, \\ 38 Fletcher Place, \\ Burlington, VT \\ 05401-1419, USA \\ $\mathrm{J}$ R Hughes}

Correspondence to: John R Hughes, MD
Harm reduction strategies applied to illicit drug dependence and examples in applying them to smoking

Increase ease of access to treatment (for example, provide smoking cessation treatment as part of routine medical services)

Use safer delivery routes (for example, low tar cigarettes)

Decrease drug intake in non-abstainers (for example, decrease cigarettes/d)

Monitor for early detection of associated diseases/problems (for example, regular $x$ rays for lung cancer)

Protect non-users (for example, restrict places where smoking allowed)

Destigmatise use (for example, create empathy for those unable to stop smoking) 


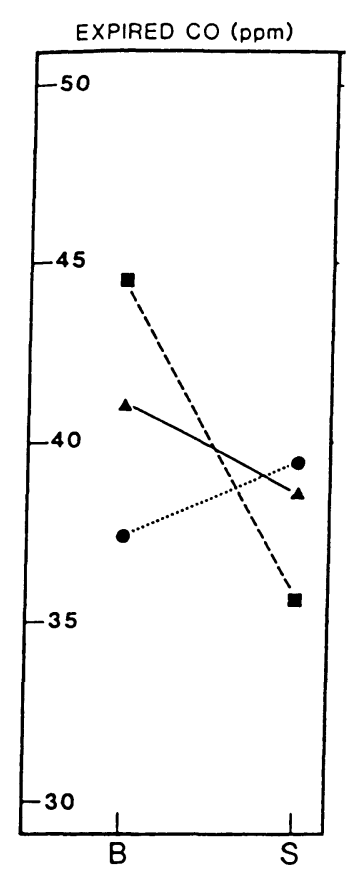

Figure 1 Expired carbon monoxide levels in smokers who decreased (boxes), increased (circles), or did not change (triangles) the nicotine yield of their cigarettes from baseline $(B)$ to the 3-6 year follow up study $(S)$. Reprinted with permission from reference 10.

the market in recent years. ${ }^{5,21,22}$ However, very low tar cigarettes $(<6 \mathrm{mg}$ tar per cigarette) continue to make up less than $10 \%$ of the market despite large advertising expenditure. ${ }^{22}$ This may be because the taste of cigarettes is highly influenced by tar levels, ${ }^{5}$ or because very low nicotine cigarettes fail to produce sufficient nicotine to abate withdrawal symptoms. ${ }^{23}$

Another concern is that smokers are misled by the use of numerical descriptions of tar levels. $^{24,25}$ For example, some smokers may believe a decrease in tar yield from $15 \mathrm{mg}$ to $5 \mathrm{mg}$ per cigarette would produce a threefold reduction in mortality. In all likelihood, this is not true. ${ }^{5}$ In fact, existing surveys suggest that smokers rely overmuch on the tar numbers and overestimate the benefit of low tar cigarettes. ${ }^{24}$

On the basis of these concerns, the President's Cancer Panel included the following suggestions. A simple graphic representation should be provided with each pack of cigarettes sold in the USA and in all advertisements. The representation should not imply a one to one relationship between measurements and disease risk. The system must be accompanied by public education to make smokers aware that individual exposure depends on how the cigarette is smoked and that the benefits of switching to lower yield cigarettes are small compared to quitting. ${ }^{4}$

Finally, there is the concern over whether encouraging those not interested in quitting to switch to low tar, low nicotine cigarettes would increase or decrease the likelihood that they would later stop smoking. Switching might increase cessation, since with successful switching to low tar cigarettes smokers experience some success over controlling their smoking behaviour and this could encourage a later full attempt at cessation. In addition, there is some evidence that switching to a low tar, low nicotine cigarette would decrease nicotine dependence ${ }^{26,27}$ and thereby increase cessation rates.

On the other hand, switching might decrease the probability of future cessation, as smokers who switch rationalise that they have done something to decrease the harmful effects of smoking and this rationalisation decreases their perceived need to stop. In fact, in one recent study a firm commitment to complete abstinence was the most powerful predictor of long term abstinence ${ }^{28}$; thus anything that decreases commitment to abstinence might undermine cessation efforts.

The only relevant data on the effects of switching to low tar cigarettes on subsequent quitting are from the study on smokers not wishing to stop, described above. ${ }^{9,20}$ In this study, at the 2.5 year follow up $9 \%$ had stopped smoking. Since there was no matched control group, it is unclear whether this cessation rate is more or less than would occur in a similar group of non-switchers. Finally, any benefits of low nicotine, low tar cigarettes might be negated by increased initiation of smoking because of the low nicotine; however, whether the availability of low nicotine cigarettes increases rates of taking up smoking is unclear. $^{1,2}$

\section{Less hazardous products: low tar, medium nicotine cigarettes}

Since the increased smoking of low tar, low nicotine cigarettes is caused by nicotine seeking, ${ }^{1,2}$ one proposal is to develop low tar, medium nicotine cigarettes. ${ }^{29-31}$ Although tar and nicotine yields are highly related, there is some variance in tar/nicotine ratios. For example, in 1988, brands of Gauloise and John Player cigarettes both had tar yields of $15 \mathrm{mg}$ but the former had a nicotine yield of $0.86 \mathrm{mg}$ per cigarette and the latter a yield of $1.44 \mathrm{mg}$ per cigarette. ${ }^{29}$ Given that nicotine seeking occurs, the latter should be smoked less intensely than the former and deliver a lower tar intake. Unfortunately, the possible advantages of a low tar, medium nicotine cigarette have not been researched.

\section{Less hazardous products: commercial nicotine products}

Another strategy is to use products that deliver only nicotine. This raises the issue of the relative harm from nicotine in cigarettes. Tar and carbon monoxide have been deemed to be causal agents in smoking related diseases; however, according to a recent Surgeon General's report on smoking and health, "nicotine may contribute to the pathogenesis of smoking related diseases, although direct causation has not been determined." 32 In fact, some lines of evidence suggest that nicotine is not a causal agent. ${ }^{33,34}$

Two commercial devices that deliver nicotine without burning tobacco have recently been tested. ${ }^{35-37}$ Although both claim to use tobacco material to deliver nicotine, some 
believe these are actually true nicotine delivery devices. ${ }^{38}$ Two studies have reported that they do indeed deliver almost no $\operatorname{tar}(<1 \%)$ and as a result tar levels decline dramatically in people who switch to them (for example, by $90 \%)^{35,39,40}$ Nicotine levels also declined somewhat in one study $(-30 \%)^{40}$ but not in the other..$^{39}$ More importantly, when smokers switched to the device, carbon monoxide levels increased somewhat in one study $(+18 \%)^{40}$ Since the increase in carbon monoxide is small compared to the decrease in tar, one might assume that these devices would reduce overall health risk; however, carbon monoxide is associated with tobacco induced cardiovascular disease, which is a more common cause of smoking induced deaths than cancers. ${ }^{41}$ Thus acceptance of these devices as safer will require more testing. There is little published data on the acceptability of these devices; however, the first of the two devices was recalled, in part because of consumer dissatisfaction. Finally, as with low tar cigarettes, whether using such devices would increase or decrease the probability of future cessation or initiation of smoking is unknown.

\section{Less hazardous products: pharmaceutical nicotine products}

The other existing non-tobacco delivery systems are nicotine reduction medications: gum patch, nasal spray, and inhaler. Although these are marketed for use as cessation aids, in the case of nicotine gum at least, substantial numbers of smokers use them in the long term to relieve withdrawal caused by smoking restrictions or to decrease the amount of smoking. ${ }^{4,43}$ Such behaviours are especially likely to occur if these products go over-thecounter. Short term studies suggest no significant adverse cardiovascular effects from gum and patch. ${ }^{33,34}$ One adverse effect from long term use is a continuance of nicotine dependence ${ }^{42}$; however, how harmful this would be in itself is unclear. Given the absence of carcinogens and carbon monoxide and the low nicotine blood level in long term gum and patch users, ${ }^{42}$ the risk of long term use of these aids appears minimal. ${ }^{42}$ On the other hand, early data suggest that the long term use of nicotine nasal spray may produce substantial nicotine levels ${ }^{44}$; thus the safety of nasal spray used under these conditions will need more testing. As with low tar cigarettes, there are no data on whether the use of nicotine medications with a non-cessation goal of reducing smoking increases or decreases the probability of later quitting.

\section{Reducing intake: number of cigarettes smoked/day}

The risks of smoking are strongly dose related; for example, in one study the risk of lung cancer increased from 10 -fold in those who smoked 1-10 cigarettes per day to 40-fold in those who smoked 21-30 cigarettes per day, and to 70 -fold in those who smoked 40 or more cigarettes per day (figure 2). ${ }^{45}$ However, these

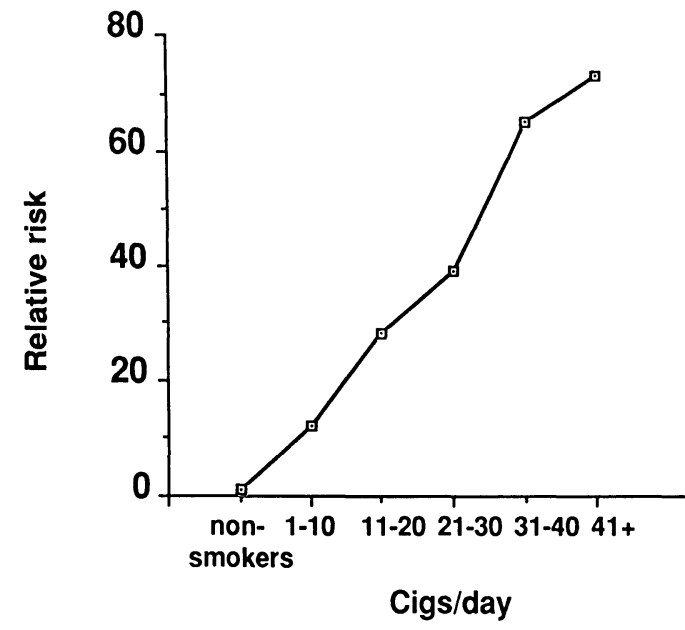

Figure 2 Relative risk of lung cancer in male smokers of filtered cigarettes by number of cigarettes smoked/d. Reprinted with permission from reference 45.

risk figures are based on smokers who self selected their rate of smoking. As with low tar cigarettes, neither prospective studies of those who reduce their number of cigarettes per day nor randomised trials of reducing cigarettes per day are available. The existing data on reducing the number of cigarettes per day outlined below come from three different populations: smokers who tried abrupt cessation and failed and instead resorted to smoking fewer cigarettes; smokers given a reduction programme before cessation; and smokers who were not interested in quitting. Results from the first two groups may not be applicable to the third group, given the very different goals of the populations.

Among smokers who tried to stop but were unable to and instead reduced their number of cigarettes per day, the large majority never achieve abstinence. ${ }^{46}$ However, many maintain significant reductions in the number of cigarettes per day for long periods of time. ${ }^{47}$

Behavioural interventions $s^{17,18,20,48}$ have been successful in decreasing the number of cigarettes per day as a preparation stage for smokers who wish to quit. Some of these studies describe a "stuck point" of 8-10 cigarettes per day below which reducing the number is very difficult. ${ }^{49}$ This observation is plausible as it may be that only a few cigarettes per day are needed to abate nicotine withdrawal, and this stuck point is where withdrawal is elicited. ${ }^{1}$ The other possibility is that at this low dose, nicotine serves to prime greater drug intake rather than to decrease drug intake..$^{50}$

There are but two studies of reduced smoking in those without abstinence as a goal. In the controlled smoking study referred to above, smokers reduced from 34 cigarettes per day before treatment to 21 cigarettes per day after treatment and to 26 cigarettes per day at the 2.5 year follow up. ${ }^{9,20}$ In the other study, smokers randomised to $2 \mathrm{mg}$ or $4 \mathrm{mg}$ nicotine gum or placebo reduced from $43-44$ to $19-22$ cigarettes per day at a six month follow up, with no difference across groups. ${ }^{51}$

One might doubt that benefit from a reduction in the number of cigarettes occurs, given that in the short term smokers who 


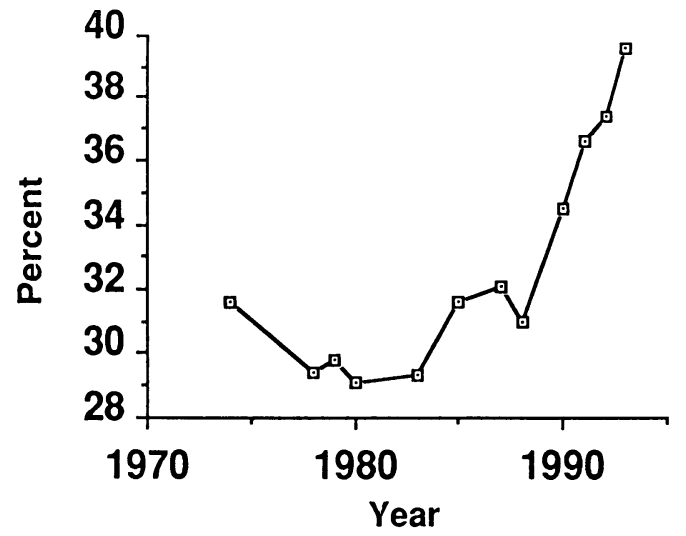

Figure 3 Percentage of current cigarette smokers who smoke less than 15 cigarettes/d (Centers for Disease Control and Prevention, personal communication).

decrease the number of cigarettes per day increase the intensity of smoking each cigarette. ${ }^{1-3}$ Unfortunately, few studies of the use of low tar cigarettes or of decreasing the number of cigarettes per day reported biochemical values to examine this issue. Among smokers who could not quit and resorted to less frequent smoking, one study $\operatorname{did}^{47}$ and one did not $^{52}$ find reductions in carbon monoxide or thiocyanate at follow up. Among smokers not trying to quit, both studies found carbon monoxide decreased by $9 \mathrm{ppm}$ at follow up in those who reduced the number of cigarettes per day. ${ }^{20,51}$

The incidence of low rate smoking (that is, $<15$ cigarettes per day) has increased dramatically in the last decade (figure 3). One explanation for this change is the implementation of public policies to restrict where one can smoke. The biggest effect of such restrictions is not to increase cessation but rather to decrease the ongoing rate of smoking. ${ }^{53}$ For example, in one study, only $6 \%$ of smokers quit but $54 \%$ decreased their smoking by an average of $40 \%$ from their baseline number of cigarettes per day. ${ }^{54}$

\section{Reducing intake: smoking topography}

The other method of decreasing intake is to change how one smokes a cigarette (for example, decrease the number of puffs per cigarette, puff duration, or puff volume). Smokers can be taught to change their smoking topography; however, whether these changes can be maintained over time is unclear. ${ }^{9.55}$ For example, in the previously cited study of smokers without abstinence as a goal, the percent of the cigarette smoked was $85 \%$ initially, declined to $62 \%$ after treatment, but increased back to $79 \%$ at a 2.5 year follow up. ${ }^{20}$ In addition, the data showing that the risks of smoking are related to smoking topography are weak. ${ }^{56}$

\section{Possible harm reduction interventions}

The sections above have mentioned behavioural, pharmacological, and public health interventions to help smokers reduce their smoking. Two other possible interventions are a simple increase in taxation on cigarettes or taxation based on tar levels. Although most of the data suggest that taxation has its greatest effects on initiation, most studies have shown some effect on decreasing the number of cigarettes per day among continuing smokers. ${ }^{57}$

In terms of interventions focused on individuals, it is unclear when reductions in smoking should be advocated. Some would use a reduction goal only after several serious attempts at cessation had failed. Others might suggest reduction as a treatment for precontemplators or contemplators not ready to stop smoking ${ }^{58}$ but willing to do something, in the hope that this half step would later lead to cessation. Whether rules often used in controlled drinking (for example, spouse approval, no substance induced disease, no physical dependence $)^{59}$ would translate to smoking is unclear.

\section{Summary}

In conclusion, there are insufficient data to conclude that smoking harm reduction is beneficial and should be pursued; however, this area is highly deserving of study. The rationale for this conclusion is as follows. It appears that switching to low tar cigarettes, reducing the number of cigarettes per day, and the long term use of nicotine-only products can be maintained, and backsliding and compensatory changes do not negate these efforts. Although switching to low tar cigarettes and smoking fewer cigarettes per day appear to offer some health benefit, this benefit is not well proven and is small compared to that of cessation. On the other hand, long term use of commercial or pharmaceutical nicotine products appears to produce relatively small doses of nicotine, few if any carcinogens, and, in the case of medications, no carbon monoxide. Despite this favourable profile, there are no studies of the long term risks and benefits of these products.

Most importantly, the major issue with all these harm reduction strategies is whether, when applied to smokers who do not presently wish to quit, they increase or decrease the probability of later smoking cessation. If these strategies undermine smokers' resolve to stop smoking, then any benefits of reduced intake would have to be compared to the harm caused by decreased cessation. If, on the other hand, these strategies either increased or did not change smoking cessation, then smokers might be encouraged to undertake harm reduction, with the caveat that smokers understand that the expected benefit may be small and is not completely proven.

\section{Research needs}

Among the many questions that need to be investigated on applying harm reduction to smoking, five seem especially important:

(1) Does switching to low tar cigarettes or reducing the number of cigarettes per day improve health? A randomised trial of the 
effects of low tar cigarettes (with either medium or low nicotine yields) or reduced number of cigarettes on health outcomes would be ideal but, given the large number of subjects needed, would be very expensive. As an alternative, several databases could be used to examine whether smokers who switched to low tar cigarettes or reduced their smoking had less morbidity and mortality than smokers who did not engage in these changes.

(2) Would a low tar medium nicotine cigarette produce less exposure to carcinogens and carbon monoxide than a low tar low nicotine cigarette?

(3) What are the health benefits and toxicity of long term use of nicotine-only delivery devices or medications?

(4) Among smokers who reduce the number of cigarettes either to reduce health risk or because of work site restrictions, according to biochemical indicators how well do they maintain such reductions and to what degree are these reductions negated by compensatory smoking?

(5) Among smokers who do not wish to stop smoking, does switching to low tar cigarettes, or switching to nicotine-only products, or reducing the number of cigarettes per day increase or decrease the probability of later stopping smoking? As above, if this question could not be investigated with a randomised trial, then a prospective study of smokers who switched or reduced their smoking would be valuable. To me, this is the most important question to be answered.

1 Kozlowski LT. Reduction of tobacco health hazards in continuing users: individual behavioral and public health continuing users: individual behavioral and publ

2 Kozlowski LT. Less-hazardous tobacco use as a treatment for the smoking and health problems. In: Smart RJ, Cappell HD, Glaser F, Israel Y, Kalant H, Schmitt W, et al, eds. Research advances in alcohol and drug problems. New York: Plenum, 1984: 309-28.

3 Benowitz NL. Health and public policy implications of the "low yield" cigarette. N Engl F Med 1989; 320: 1619-21.

4 National Cancer Institute. FTC cigarette test method. A report of the NCI expert panel. Smoking and Tobacco Control Monograph No 8. Bethesda, Maryland: National Cancer Institute (in press)

5 US Department of Health and Human Services. The health consequences of smoking : the changing cigarette : a report of
the Surgeon General. Rockville, Maryland: Public Health the Surgeon General. Rockville, Maryland: Public Health and Health, 1981. (DHHS Publication No. (PHS) 81and Health.)

6 Hammond EC, Garfinkel L, Seidman H, Lew EA. "Tar" and nicotine content of cigarette smoke in relation to death rates. Environ Res $1976 ; 12$ : 263-74.

7 Kaufman DW, Palmer JR, Rosenberg L, Stolley P, Warshauer E, Shapiro S. Tar content of cigarettes in relation to lung cancer. Am 7 Epidemiol 1989; 129: 703-10

8 DeGrandpre RJ, Bickel WK, Hughes JR, Higgins ST. Behavioral economics of drug self-administration. III : A reanalysis of the nicotine regulation hypothesis. Psychopharmacology 1992;108: 1-8.

pharmacology 1992; 108: 1-8.
9 Glasgow RE, Klesges RC, Klesges LM, Vasey MW, Gunnarson DF. Long-term effects of a controlled smoking program: a $2 \frac{1}{2}$ year follow-up. Behav Ther 1985 ; 16: 303-7.

10 Lynch CJ, Benowitz NL. Spontaneous cigarette brand switching: consequences for nicotine and carbon monoxide exposure. Am F Public Health 1987; 78: 1191-4.

11 Guyatt AR, Kirkham AJT, Mariner DC, Baldry AG, Cumming $G$. Long-term effects of switching to cigarettes with lower tar and nicotine yields. Psychopharmacology $1989 ; 99: 80-6$

12 Kanzler M, Jaffee JH, Nee J. Low nicotine cigarettes: cigarette consumption and breath carbon monoxide after one year. Clin Pharmacol Ther 1983; 34: 408-15.

13 Foxx RM, Brown RA. Nicotine fading and self-monitoring for cigarette abstinence or controlled smoking. $₹ \mathrm{Appl}$ Behav Anal 1979; 12: 111-25.

14 Foxx RM, Axelroth E. Nicotine fading, self-monitoring and cigarette fading to produce cigarette abstinence or controled smoking. Behav Res Ther 1983; 21: 17-27.

15 Beaver C, Brown RA, Lichtenstein E. Effects of monitored nicotine fading and anxiety management training on smoking reduction. Addict Behav 1981; 6: 301-5.

16 Colletti G, Supnick JA, Rizzo AA. Long-term follow-up (3-4 years) of treatment for smoking eduction. Addict Behav 1982; 7: 429-33.

17 Reynolds RVC, Tobin DL, Creer TL, Wigal JK, Wagner MD. A method for studying controlled substance use: a
preliminary investigation. Addict Behav 1987; 12:53-62.

18 Becona E, Garcia MP. Nicotine fading and smokeholding methods to smoking cessation. Psychol Rep 1993; 73: $779-86$

19 Lando HA, McGovern PG. Nicotine fading as a nonaversive alternative in a broad-spectrum treatment for eliminating smoking. Addict Behav 1985; 10: 153-61.

20 Glasgow RE, Klesges RC, Godding PR, Gegelman R. Controlled smoking, with or without carbon monoxide feedback, as an alternative for chronic smokers. Behav Ther 1983; 14: 386-97.

21 Federal Trade Commission. Federal Trade Commission report to Congress. Washington, DC: US Government Printing Office: 1995

22 Kozlowski LT. Evidence for limits on the acceptability of lowest-tar cigarettes. Am $\mathcal{F}$ Public Health 1989; 79: 198-9.

23 West RJ, Russell MAH, Jarvis MS, Feyerabend C. Does switching to an ultra-low nicotine cigarette induce nicotine withdrawal effects? Psychopharmacology 1984; 84: $120-3$.

24 Chapman S, Wilson D, Wakefield M. Smokers' understandings of cigarette yield labels. Med $\mathcal{F}$ Aust $1986 ; 145$ : 376-9.

25 Henningfield JE, Kozlowski LT, Benowitz NT. A proposal to develop meaningful labeling for cigarettes. $\mathcal{F} A M A$ 1994; 272: 312-4.

26 Benowitz NL. Establishing a nicotine threshold for addiction. $N$ Engl f Med 1994; 331: 123-5.

27 Hughes JR. Regulation of the nicotine content of cigarettes. N Engl f Med 1994; 331: 1530-1.

28 Hall SM, Havassy BE, Wasserman DA. Effects of commitment to abstinence, positive moods, stress and coping on relapse to cocaine use. $\mathcal{F}$ Consult Clin Psychol 1991; 59 526-32.

29 Russell MAH. Realistic goals for smoking and health : a case for safer smoking. Lancet 1974; i: 254-7.

30 Stepney R. Would a medium-nicotine, low-tar cigarette be less hazardous to health? $B M \mathcal{F} 1981 ; 283: 1292-6$.

31 Russell MAH. Low-tar medium-nicotine cigarettes: a new approach to safer smoking. $B M \mathcal{F}$ 1976; i: $1430-3$.

32 US Department of Health and Human Services. The health consequences of smoking : nicotine addiction. A report of the Surgeon General, 1988. Rockville, Maryland: Public Health Service, Centers for Disease Control, Office on Health Service, Centers for Disease Control, Office on
Smoking and Health, 1988. (DHHS Publication No (CDC) 88-8406.)

33 Benowitz NL. Pharmacologic aspects of cigarette smoking and nicotine addiction. NEngl f Med 1988; 319: 1318-30

34 Hughes JR. Risk/benefit of nicotine replacement in smoking cessation. Drug Safety 1993; 8: 49-56.

35 Hilts PJ. Little smoke, little tar, but still lots of nicotine. New York Times (National), Nov 27, 1994: 1 .

36 deBethizy JD, Borgerding MF, Doolittle DJ, Robinson JH, McManus KT, Rahn CA, et al. Chemical and biological studies of a cigarette that heats rather than burns tobacco. $\mathcal{f}$ Clin Pharmacol 1990; 30: 755-63.

37 Hwant SL. Library search hints of electrifying news from big tobacco. Wall Street fournal, Feb 6, 1995 .

38 Slade J, Connolly GN, Davis RM, Douglas CE, Henningfield JE, Hughes JR, et al. Report of the tobacco policy research study group on tobacco products. Tobacco Control 1992; 1: 54-9.

39 Woodson PP, Fagerstrom K, Molander L, Slade J, Henningfield JE. Premier: a new nicotine delivery system - studies on its environmental and biological impact. $f$ Smoking Related Dis 1993; 4: 191-201.

40 Sutherland G, Russell MAH, Stapleton JA, Feyerabend C. Clycerol particle cigarettes: a less harmful option for chronic smokers. Thorax 1993; 48: 385-7.

41 US Department of Health and Human Services. The health benefits of smoking cessation. A report of the Surgeon
General, 1990. Rockville, Maryland: Public Health Service, Centers for Disease Control, Office on Smoking and Health, 1990. (DHHS Publication No (CDC) a0-8416.)

42 Hughes JR. Dependence potential and abuse liability of nicotine replacement therapies. Biomed Pharmacother nicotine replace

43 Johnson RE, Stevens VJ, Hollis JF, Woodson GT. Nicotine chewing gum use in the outpatient care setting. $\mathcal{F}$ Fam Pract 1992; 34: 61-5.

44 Sutherland G, Stapleton JA, Russell MAH, Jarvis MJ Hajek P, Belcher $M$, et al. Randomised controlled trial of nasal nicotine spray in smoking cessation. Lancet 1992 340: 324-9.

45 Wynder EL, Stellman SD. Impact of long-term filter cigarette usage on lung and larynx cancer risk: a casecontrol study. I Natl Cancer Inst 1979; 62: 471-7.

46 Hill D, Weiss DJ, Walker DL, Jolley D. Long-term evaluation of controlled smoking as a treatment outcome. Br ₹ Addict 1988; 83: 203-7.

47 Norregaard J, Tonnesen P, Simonsen K, Petersen L, Sawe U. Smoking habits in relapsed subjects from a smoking 
cessation trial after one year. $B r f$ Addict 1992; 87: 1189-94

48 Cinciripini PM, Lapitsky LG, Wallfisch A, Mace R, Nezami E, Van Vunakis $H$. An evaluation of a multicomponent treatment program involving scheduled smoking and relapse prevention procedures: Initial findings. Addict Behav 1994; 19: 13-22.

49 Shapiro D, Schwartz GE, Tursky B, Shnidman SR. Smoking on cue: a behavioral approach to smoking Smoking on cue: a behavioral approach to smok

50 Fant RV, Gross J, Stitzer ML. Effects of smoking exposure on subsequent desire to smoke. Poster presented at the on subsequent desire to smoke. Poster presented at the
annual meeting of Society of Behavioral Medicine, San

51 Rennard SI, Daughton DM, Thompson AB, Floreani AA, Romberger DJ, Millatmal T. The effects of nicotine replacement therapy on cigarette smoking reduction. Abstract presented at the American Lung Assocation, American Thoracic Assocation Conference, Boston, MA, May, 1994.

52 Hughes GH, Hymowitz N, Ockene JK, Simon N, Vogt TM. The multiple risk factor intervention trial (MRFIT). Prev Med 1981; 10: 476-500.

53 US Department of Health and Human Services. Reducing the health consequences of smoking: 25 years of progress. A report of the Surgeon General. Rockville, Maryland: Public Health Service, Centers for Disease Control, Office on Smoking and Health, 1989. (DHHS Publication No (CDC) 89-8411.)

54 Baile WF, Gibertini M, Ulschak F, Snow-Antle S, Hann D. Impact of a hospital smoking ban: changes in tobacco use and employee attitudes. Addict Behav 1991; 16: 419-26.

55 Frederiksen LW, Peterson GL, Murphy WD Controlled smoking: development and maintenance. Addict Behav 1976; 1: 193-6.

56 US Department of Health and Human Services. The health consequences of smoking: cancer. A report of the Surgeon General. Rockville, Maryland: Public Health Service, Centers for Disease Control, Office on Smoking and Centers for Disease Control, Office on Smoking and
Health, 1982. (DHHS Publication No. (PHS) 82-50179.) 57 Townsend J, Roderick P, Cooper J. Cigarette smoking by socioeconomic group, sex, and age: effects of price, income, and health publicity. BMF 1994; 309: 923-7.

58 Prochaska JO, Goldstein MG. Process of smoking cessation: implications for clinicians. Clin Chest Med 1991; 12: $727-35$.

59 Sobell LC, Sobell MB, Nirenberg TD. Behavioral assessment and treatment planning with alcohol and drug abusers : a review with an emphasis on clinical application. Clin Psychol Rev 1988; 8: 19-54.

\title{
Panel discussion
}

\section{Clinical \\ Pharmacology \\ Branch, National \\ Institute on Drug \\ Abuse, Addiction \\ Research Center, \\ Baltimore, Maryland, \\ USA \\ J E Henningfield}

Mayo Nicotine

Dependence Center,

Mayo Clinic,

Rochester, Minnesota,

USA

R D Hurt

Department of

Medicine, Johns

Hopkins University,

Baltimore, Maryland

C S Rand

National Institute on

Drug Abuse,

Rockville, Maryland,

USA

F J Vocci

\author{
Moderator: Jack E Henningfield \\ Panellists: Richard D Hurt, Cynthia S Rand, Frank J Vocci
}

\section{Jack E Henningfield}

I am going to put Dr Vocci on the spot. Frank, you are one of the coauthors of the Guidelines for medications development which tried to level the playing field. With what you have heard today, what do you think about harm reduction with respect to tobacco?

\section{Frank J Vocci}

In the opiate field, we have somewhat acquiesced with the idea of total abstinence for all opiate users. If we can get people into treatment, we know that a certain proportion will stop using opiates, but then there is a subgroup that will not, but will reduce their use. We have somehow stuck with them and have started to accept those patients more.

I think if you use that type of a strategy, you have to start looking at these partial responders as people who are possibly giving the best response that they can. If you are conducting clinical trials in smoking cessation, you could look at secondary indications, like reduction of use, because for every drug that produces cessation, there is going to be a subgroup with reduced use. This subgroup is probably embedded within the clinical trial. All you need to do is to analyse the data a little differently and do a secondary analysis. We have categorised these people originally as failures, and perhaps we just have to start thinking differently: if you can get a sustained reduction of whatever the determined magnitude is for a long enough period of time, you should be able to show a reduction in harm.

There is a tension developing between these types of proximal endpoint and some of the things I am hearing lately from Robert Temple at the FDA, who feels that perhaps we should be looking at different endpoints. The other day he made the analogy of the congestive heart failure trials, saying that instead of looking at cardiovascular indices such as ejection fraction improvement we should perhaps be doing large scale clinical trials which would show the hospital admission rate in people on a particular treatment, and how soon these people die from congestive heart failure. Perhaps we should look at these very gross endpoints, and look at them in large populations rather than doing sophisticated modellings that may or may not show harm reduction in the disease process. It is one of the issues FDA is concerned about in clinical trials - you can have a very fancy clinical measure that does not necessarily translate to patient benefit in the long term

Philosophically, I sympathise with this concept of harm reduction. I do not think you are going to get everyone who is a smoker to stop smoking, and I think we have to start thinking about what the magnitude and duration of a reduction are that will produce a meaningful benefit in whatever indication we are looking at, whether it is a reduction of cardiovascular, pulmonary, or cancer risk.

\section{Jack E Henningfield}

Dr Rand, the lung health study you worked on had facets that might be construed as harm reduction approaches in so far as gum was relatively freely available for a long time. What are the pros or cons from your perspective?

\section{Cynthia S Rand}

To orient those who may be unfamiliar with it, the lung health study, for which final results were published in $\mathcal{F} A M A$ in November 1994, enrolled 6000 people with early chronic ob- 